\title{
QUALIDADE DE VIDA DOS ENFERMEIROS DAS EQUIPES DE SAÚDE DA FAMÍLIA: A RELAÇÃO DAS VARIÁVEIS SOCIODEMOGRÁFICAS ${ }^{1}$
}

\author{
Janielle Silva Fernandes², Sybelle de Souza Castro Miranzi $i^{3}$. Helena Hemiko Iwamoto ${ }^{4}$, Darlene Mara dos \\ Santos Tavares ${ }^{5}$ Claudia Benedita dos Santos ${ }^{6}$
}

${ }^{1}$ Trabalho decorrente de pesquisa financiada pela Fundação de Amparo à Pesquisa do Estado de Minas Gerais.

2 Mestre em Atenção à Saúde pelo Programa de Pós-Graduação Stricto Sensu em Atenção à Saúde da Universidade Federal do Triângulo Mineiro (UFTM). Minas Gerais, Brasil. E-mail: janebiju@hotmail.com.br

${ }^{3}$ Doutora em Enfermagem em Saúde Pública. Professora Adjunto do Departamento de Medicina Social da UFTM. Minas Gerais, Brasil. E-mail: sybelle@mednet.com.br

${ }^{4}$ Doutora em Enfermagem Fundamental. Professora Adjunto do Departamento de Enfermagem em Assistência Hospitalar da UFTM. Minas Grais, Brasil. E-mail: helena.iwamoto@gmail.com

${ }^{5}$ Doutora em Enfermagem. Professora Adjunto do Departamento de Enfermagem em Educação e Saúde Comunitária do Curso de Graduação em Enfermagem da UFTM. Minas Gerais, Brasil. E-mail: darlenetavares@netsite.com.br

${ }^{6}$ Doutora em Estatística. Professora Adjunto do Departamento de Enfermagem Materno-Infantil e Saúde Pública da Escola de Enfermagem de Ribeirão Preto, Universidade de São Paulo (USP). São Paulo, Brasil. E-mail: cbsantos@eerp.usp.br

RESUMO: Objetivou-se conhecer a qualidade de vida dos enfermeiros que compõem as equipes de saúde da família da Macrorregião de Saúde do Triângulo Sul, assim como fatores sociodemográficos relacionados. Trata-se de um estudo descritivo e transversal. O universo, composto por 90 enfermeiros, respondeu a um questionário cuja finalidade foi conhecer as variáveis sociodemográficas influentes e o instrumento genérico para avaliar a qualidade de vida WHOQOL-100. Os resultados indicam que indivíduos casados/ amasiados obtiveram maiores escores de qualidade de vida comparados aos solteiros, nos domínios psicológico $(p=0,013)$, independência $(p=0,021)$, relações sociais $(p=0,000)$ e ambiente $(p=0,022)$. Aqueles que declararam saúde debilitada apresentaram menores escores nos domínios físico $(p=0,000)$, psicológico $(p=0,002)$ e independência $(p=0,000)$. Os resultados mostram impacto negativo do estado de saúde debilitado nos domínios da qualidade de vida dos enfermeiros. Ressalta-se a importância da promoção de saúde e considera-se necessária a (re)definição de políticas públicas voltadas para a saúde desses profissionais.

DESCRITORES: Qualidade de vida. Saúde da família. Saúde do trabalhador. Recursos humanos em saúde. Enfermagem.

\section{QUALITY OF LIFE OF FAMILY HEALTH TEAM NURSES: THE RELATIONSHIPS OF SOCIODEMOGRAPHIC FACTORS}

\begin{abstract}
This study aimed better understand the Quality of Life among nurses who make up the family health teams of the Brazilian Health Macro-region of its Southern Triangle, as well as related socio-demographic factors. This is a descriptive cross-sectional study. The universe, composed of 90 nurses, answered a questionnaire whose purpose was to identify influential socio-demographic variables and the generic instrument in assessing its WHOQOL-100 Quality of Life. The results indicate that compared to single people, married individuals had higher Quality of Life scores in psychological $(p=0.013)$, independence $(p=0.021)$, social $(p=0.000)$, and environment $(p=0.022)$. Those who reported poor health had lower scores in physical $(p=0.000)$, psychological $(p=0.002)$, and independence $(p=0.000)$. The results show the negative impact of the weakened state of health in the areas of Quality of Life of nurses. This emphasizes the importance of health promotion, with the (re)definition of public policies for their Brazilian health professionals considered a necessity.
\end{abstract}

DESCRIPTORS: Quality of life. Family health. Occupational health. Health manpower. Nursing.

\section{LA CALIDAD DE VIDA DE LAS ENFERMERAS DE LOS EQUIPOS DE SALUD FAMILIAR: LA RELACIÓN DE VARIABLES SOCIODEMOGRÁFICAS}

\begin{abstract}
RESUMEN: Este estudio, descriptivo y transversal, tuvo como objetivo conocer la calidad de vida de las enfermeras que conforman los equipos de salud familiar de la Macroregión de Salud del Triángulo Sur, así como los factores sociodemográficos relacionados. La muestra se formó con 90 enfermeras, quienes respondieron a un cuestionario cuyo finalidad fue identificar las variables sociodemográficas influyentes y el instrumento genérico para evaluar la calidad de vida WHOQOL-100. Los resultados indican que los individuos casados tenían puntuaciones más altas en comparación con la calidad de vida de los solteros, en el aspecto psicológico $(p=0,013)$, independencia $(p=0,021)$, social $(p=0,000)$ y el ambiente $(p=0,022)$. Aquellos que informaron mala salud tenían puntuaciones más bajas en lo físico $(p=0,000)$, psicológico $(p=0,002)$ e independencia $(p=0,000)$. Los resultados muestran el impacto negativo del estado de salud en las áreas relacionadas con la calidad de vida de las enfermeras. Se hace hincapié en la importancia de promover la salud y se considera necesaria la (re)definición de políticas públicas para los profesionales de la salud.
\end{abstract}

DESCRIPTORES: Calidad de vida. Salud de la família. Salud laboral. Recursos humanos en salud. Enfermería. 


\section{INTRODUÇÃO}

Nos últimos anos, a Estratégia Saúde da Família (ESF), como política pública nacional, tem-se destacado como modelo estratégia de reorganização da atenção básica fundamentada baseada na lógica da vigilância à saúde. Foi implantada em todo o Brasil como o principal e mais importante meio para a reordenação da atenção à saúde, conforme preconizam os princípios e diretrizes do Sistema Único de Saúde. Suas ações representam a concepção de saúde centrada na promoção da Qualidade de Vida (QV), por meio dos seus principais objetivos, que são: a prevenção, a promoção e a recuperação da saúde. ${ }^{1}$

Entender a Saúde da Família como estratégia de mudança baseada na reorientação do modelo de atenção à saúde significa repensar as práticas, os valores e os conhecimentos de todas as pessoas envolvidas no processo de produção social da saúde. Amplia-se a complexidade das ações a serem desenvolvidas e aumentam-se os limites e possibilidades de atuação, o que requer dos profissionais de saúde novas habilidades.

Os profissionais precisam desenvolver um trabalho de qualidade, capaz de estimular estimulando a comunidade na busca de melhores condições de saúde. Desta forma, devem visualizar diversos aspectos que envolvem a população, incluindo as condições biopsicossociais, econômicas, culturais e espirituais. Para que isto ocorra, entende-se que o profissional de saúde precise de $\mathrm{QV}$, já que os fatores que nela interferem podem comprometer sobremaneira a qualidade do cuidado prestado.

Conhecer a QV desses trabalhadores permite identificar as mudanças necessárias para a promoção do bem-estar, assim como a adequação de condições para a sua reabilitação. Dependendo dos domínios afetados na QV, os mesmos trabalhadores podem deparar-se com diversos transtornos, inclusive com comprometimento das funções e atribuições no trabalho. ${ }^{2}$

Entre os componentes das equipes de saúde da família, esta pesquisa faz um recorte para a $Q V$ do enfermeiro, em função de sua relevância como ator social e para o desenvolvimento dos sistemas de saúde. Inserido no movimento de reordenação do modelo de atenção à saúde, ele se destaca pela significativa responsabilidade nas diversas ações desenvolvidas na comunidade e na sua equipe. de saúde ${ }^{3}$ Esses profissionais são agentes participantes e encarregados de operar as atividades básicas da ESF e as ações promovidas por eles influem diretamente no processo de implementação e resultados da estratégia. ${ }^{4-5}$

Tem-se como pressuposto que a presença de alterações físico-emocionais ou sócio-familiares e as doenças iminentes, dentre outros fatores, podem alterar o estilo de vida e afetar a QV dos enfermeiros em um curto espaço de tempo.

A preocupação em se conceituar o termo QV surgiu em meados da década de 70 e até os dias atuais não houve consenso entre os pesquisadores sobre a definição deste termo. ${ }^{6}$

As informações sobre QV têm sido utilizadas para avaliar a eficácia de determinados tratamentos de doenças, os agravos à saúde e o impacto físico e psicossocial, ${ }^{7,8}$ mas também são apropriadas a estudos epidemiológicos, planejamento e avaliação do sistema de saúde. O Grupo de QV da Organização Mundial de Saúde define o termo como: "a percepção do indivíduo de sua posição na vida, no contexto da cultura e do sistema de valores em que vive e em relação aos seus objetivos, expectativas, padrões e preocupações" ${ }^{\prime 9: 13}$

Os estudos sobre QV voltados para essa categoria profissional, no Brasil, são escassos e não possuem consenso metodológico, o que dificulta a construção de um corpus de conhecimentos específicos. Não foram encontrados estudos voltados para enfermeiros da atenção básica na literatura científica brasileira. ${ }^{4}$

A avaliação da QV dos enfermeiros oferece subsídios para melhorar o processo de trabalho em saúde, a prática clínica, a relação profissionalusuário e para orientar a (re)definição de políticas públicas específicas para esses profissionais, no desempenho de suas funções. A melhoria das condições de vida e de trabalho desses profissionais pode gerar um impacto positivo na saúde, tanto dos enfermeiros, quanto da população por eles assistida.

Neste sentido, esta investigação teve por objetivo avaliar a QV dos enfermeiros das equipes de saúde da família, verificando a relação entre as variáveis sociodemográficas e os domínios da QV.

\section{MATERIAIS E MÉTODOS}

Trata-se de um estudo descritivo e transversal. A população abordada foi composta pelos enfermeiros das equipes de saúde da família dos 27 municípios da Macrorregião de Saúde do Triângulo Sul que concordaram em participar da 
pesquisa. Foram excluídos 23 enfermeiros que se encontravam afastados temporariamente de suas atividades por motivo de licença ou em período de férias. Não houve recusa, por parte dos enfermeiros, em participar da pesquisa. Dessa forma, do total de 113 enfermeiros em exercício durante o período de coleta de dados, entre maio e julho de 2007,90 , sendo que $(79,7 \%)$ enfermeiros participaram da pesquisa.

Os dados foram coletados através de dois instrumentos autoaplicados, um para avaliação genérica da QV e outro contendo as variáveis sociodemográficas. Os participantes foram contatados nas suas respectivas Unidades de Saúde da Família, e esclarecidos sobre os objetivos da pesquisa, o preenchimento do questionário, a assinatura do Termo de Consentimento Livre e Esclarecido, a garantia do sigilo e o anonimato das respostas, e também sobre eventuais dúvidas. Somente após a anuência do entrevistado e assinatura do referido Termo conduziu-se a aplicação dos instrumentos.

Na avaliação da QV foi utilizada a versão validada no Brasil do instrumento de propriedade da Organização Mundial deSaúde, World Health Organization Quality of Life (WHOQOL-100), composto por 100 questões, distribuídas em seis domínios: físico, psicológico, nível de independência, relações sociais, meio ambiente e espiritualidade/crenças pessoais. Cada domínio é constituído por facetas, num total de 24. Cada faceta é composta por quatro perguntas. Além das 24 facetas específicas, o instrumento possui uma vigésima quinta faceta, composta de perguntas gerais sobre QV. O instrumento foi respondido em um só encontro e com base nas duas últimas semanas de vida do enfermeiro. A escolha desse questionário deveu-se ao fato de ele ser genérico, ou seja, abrangente em relação aos aspectos da QV e ter mostrado características psicométricas satisfatórias na população brasileira. ${ }^{10}$

Em relação às características sociodemográficas, o instrumento utilizado aborda as seguintes variáveis: sexo, idade, estado civil, escolaridade, curso atual, problema de saúde atual/condição de saúde presente e estado de saúde.

Os dados foram gerenciados e analisados no software SPSS 16.0. Para as variáveis categóricas utilizou-se análise descritiva a partir da apuração de frequências, tanto em termos absolutos, quanto em percentuais. Para as variáveis numéricas calcularam-se medidas descritivas de centralidade (média, mediana e moda) e de dispersão (desvio padrão, coeficiente de variação, valores máximos e mínimos). Foi empregada, ainda, a sintaxe WHOQOL-100 no SPSS para avaliação dos escores de QV. Os escores obtidos em relação aos domínios são determinados em escala de 4 a 20, em que quanto maior o valor, maior a $\mathrm{QV}$ naquele domínio. Os escores obtidos em cada domínio e em relação à QV geral foram classificados em quatro intervalos para facilitar a compreensão dos resultados (Quadro 1).

Quadro 1 - Classificação valorativa dos escores obtidos através da aplicação do instrumento WHOQOL-100 segundo análise dos intervalos proporcionais

\begin{tabular}{|c|c|l|}
\hline Escores & Intervalos proporcionais (\%) & \multicolumn{1}{c|}{ Avaliação do resultado } \\
\hline $4 \vdash 8$ & Até 25 & Maior impacto negativo dos domínios/facetas na QV \\
\hline $8 \vdash 12$ & $25 \vdash 50$ & Impacto negativo mediano dos domínios/facetas na QV \\
\hline $12 \vdash 16$ & $50 \vdash 75$ & Pouco impacto negativo dos domínios/facetas na QV \\
\hline $16 \vdash 20$ & $75+100$ & Ausência de impacto negativo dos domínios/facetas na QV \\
\hline
\end{tabular}

Foi verificada a associação das variáveis com os escores dos domínios da QV, através da comparação dos escores de QV entre as diferentes categorias das variáveis sociodemográficas, sendo utilizado o teste $\mathrm{t}$-Student para variáveis com duas categorias e ANOVA-F, seguido de comparação múltipla de Tukey para variáveis com mais de duas categorias. Nos casos em que não houve normalidade, foram utilizados os testes não paramétricos Mann-Whitney e Kruskal-Wallis, seguido de comparações múltiplas de Dunn, respectivamente. O nível de significância adotado foi de $5 \%(p<0,05)$.

Os valores de $p$ são interpretados sob a hipótese de que a casuística constitui uma amostra por conveniência de uma população hipotética com características similares. 
Esta pesquisa acompanhou os preceitos constantes na Resolução n ${ }^{0}$ 196/96 da Comissão Nacional de Ética em Pesquisa, com aprovação do Comitê de Ética em Pesquisa em Seres Humanos da Universidade Federal do Triângulo Mineiro sob o protocolo $n^{\circ} 791 / 2006$. Foi realizada com o consentimento formal dos Gestores Municipais deSaúde, através da assinatura do termo de permissão para o desenvolvimento da pesquisa, e consentimento dos participantes do estudo pela assinatura do Termo de Consentimento Livre e Esclarecido.

\section{RESULTADOS}

Entre os enfermeiros das equipes de saúde da família do Sul do Triângulo Mineiro predominou o sexo feminino $(92,2 \%)$. A idade média foi de 28,6 anos $( \pm 5,9)$, com predomínio da faixa etária de 20 30 anos (72,1\%). No que se refere ao estado civil, verificou-se que a maior parte dos profissionais era constituída pelos solteiros $(57,8 \%)$, seguidos pelos casados ou amasiados $(33,4 \%)$. Quanto à escolaridade, $60 \%$ possuíam especialização e $40 \%$ eram graduados. A maioria realizava curso de atualização no momento da entrevista $(62,2 \%)$ e 35,6\% faziam pós-graduação lato sensu.

Quanto à autopercepção da saúde, 92\% dos enfermeiros avaliaram-na como "boa ou muito boa", seguidos pelos que referiram-na como "fraca ou muito ruim" $(5,6 \%)$. Em relação à presença de alguma doença, $66,7 \%$ negaram qualquer problema de saúde.

A qualidade de vida geral, composta pelas facetas: satisfação com a vida, satisfação com a saúde, satisfação com a própria QV e avaliação da QV, obteve escore médio de 16,7, que traduz ausência de impacto negativo das facetas avaliadas na QV.

Os escores encontrados para os domínios evidenciaram médias maiores para nível de independência, aspectos espirituais/religião/crenças pessoais e relações sociais. As médias menores foram nos domínios psicológico, ambiente e físico (Tabela 1).

Tabela 1 - Escore médio, desvio padrão, mediana, valores mínimos e máximos dos escores dos domínios de qualidade de vida do WHOQOL-100 dos enfermeiros das equipes de saúde da família da Macrorregião de Saúde do Triângulo do Sul-MG, 2007. (N=90)

\begin{tabular}{lcccc}
\hline Domínios WHOQOL-100 & Mínimo & Máximo & Mediana & Escore médio (DP) \\
\hline Físico & 8,6 & 19,6 & 14,0 & $14,1(1,9)$ \\
Psicológico & 7,8 & 19,6 & 15,6 & $15,4(2,0)$ \\
Nível de independência & 12,0 & 20,0 & 17,0 & $17,0(1,6)$ \\
Relações sociais & 9,6 & 19,6 & 16,3 & $16,2(2,1)$ \\
Ambiente & 9,0 & 18,0 & 14,2 & $14,2(1,9)$ \\
Aspectos espirituais & 10,0 & 20,0 & 17,0 & $16,8(2,6)$ \\
Qualidade de vida geral & 10,0 & 20,0 & 17,0 & $16,7(2,2)$ \\
\hline
\end{tabular}

A variação obtida de 14,1 a 17,0 mostra que esses valores estão acima da faixa de neutralidade, com tendências à valoração positiva, traduzindo QV satisfatória, ou seja, pouco impacto negativo ou ausência de impacto negativo dos domínios na QV. A consistência interna do WHOQOL-100 para as facetas $(0,78)$, domínios $(0,88)$, domínios+facetas $(0,91)$ e 100 questões $(0,94)$ foi avaliada pelo coeficiente de fidedignida- de de Cronbach e atestou boa consistência interna do instrumento na população deste estudo com obtenção de valores acima de 0,70.

Não houve diferenças estatisticamente significativas entre os escores médios dos domínios de QV quando comparados entre os sexos, faixas etárias, escolaridades, cursos de atualização ou aprimoramento e doenças presentes. 
Tabela 2 - Escore médio, desvio padrão, valores mínimos e máximos dos escores das facetas de qualidade de vida do WHOQOL-100 dos enfermeiros das equipes de saúde da família da Macrorregião de Saúde do Triângulo do Sul-MG, 2007. (N=90)

\begin{tabular}{|c|c|c|c|}
\hline Facetas WHOQOL-100 & Mínimo & Máximo & Média (DP) \\
\hline \multicolumn{4}{|l|}{ Físico } \\
\hline 1. Dor e desconforto & 4,0 & 17,0 & $11,5(2,5)$ \\
\hline 2. Energia e fadiga & 8,0 & 19,0 & $13,8(2,2)$ \\
\hline 3. Sono e repouso & 8,0 & 20,0 & $16,0(2,8)$ \\
\hline \multicolumn{4}{|l|}{ Psicológico } \\
\hline 4. Sentimentos positivos & 8,0 & 20,0 & $15,7(2,2)$ \\
\hline 5. Pensar, aprender, memória e concentração & 8,0 & 20,0 & $15,2(2,3)$ \\
\hline 6. Autoestima & 8,0 & 20,0 & $16,2(2,3)$ \\
\hline 7. Imagem corporal e aparência & 5,0 & 20,0 & $15,7(3,1)$ \\
\hline 8. Sentimentos negativos & 4,0 & 17,0 & $9,9(3,1)$ \\
\hline \multicolumn{4}{|l|}{ Nível de independência } \\
\hline 9. Mobilidade & 8,0 & 20,0 & $17,0(3,0)$ \\
\hline 10. Atividades de vida cotidiana & 7,0 & 20,0 & $15,6(2,4)$ \\
\hline 11. Dependência de medicação ou de tratamento & 4,0 & 14,0 & $5,7(2,4)$ \\
\hline 12. Capacidade de trabalho & 11,0 & 20,0 & $17,1(1,9)$ \\
\hline \multicolumn{4}{|l|}{ Relações sociais } \\
\hline 13. Relações pessoais & 12,0 & 20,0 & $16,7(1,9)$ \\
\hline 14. Suporte (apoio) social & 8,0 & 20,0 & $15,5(2,5)$ \\
\hline 15. Atividade sexual & 7,0 & 20,0 & $16,3(3,1)$ \\
\hline \multicolumn{4}{|l|}{ Ambiente } \\
\hline 16. Segurança física e proteção & 5,0 & 17,0 & $12,3(2,3)$ \\
\hline 17. Ambiente no lar & 8,0 & 20,0 & $15,7(2,9)$ \\
\hline 18. Recursos financeiros & 8,0 & 20,0 & $13,0(2,6)$ \\
\hline 19. Cuidados de saúde e sociais: disponibilidade e qualidade & 6,0 & 20,0 & $14,1(2,5)$ \\
\hline 20. Oportunidades de adquirir novas informações e habilidades & 8,0 & 20,0 & $15,1(2,2)$ \\
\hline 21. Participação em/e oportunidades de recreação/lazer & 6,0 & 20,0 & $13,3(3,0)$ \\
\hline 22. Ambiente físico: (poluição/ruído/ trânsito/clima) & 9,0 & 19,0 & $14,4(2,2)$ \\
\hline 23. Transporte & 5,0 & 20,0 & $15,5(3,6)$ \\
\hline \multicolumn{4}{|l|}{ Espirituais } \\
\hline 24. Espiritualidade/religião/crenças pessoais & 10,0 & 20,0 & $16,8(2,6)$ \\
\hline
\end{tabular}

Não houve diferenças estatisticamente significativas entre os escores médios dos domínios de QV quando comparados entre os sexos, faixas etárias, escolaridades, cursos de atualização ou aprimoramento e doenças presentes.

O grupo que se declarou casado(a) ou amasiado(a) apresentou maiores escores médios de $Q V$, comparados àqueles que não possuíam companheiro(a) nos domínios psicológico $(p=0,013)$, nível de independência $(p=0,021)$, relações sociais $(p=0,000)$ e ambiente $(p=0,022)$ (Tabela 3).

O grupo que relatou possuir saúde "fraca ou muito ruim" apresentou menores escores de QV, em relação os que referiram saúde "nem ruim, nem boa" e "muito boa ou boa" nos domínios físico $(p=0,000)$, psicológico $(p=0,002)$ e nível de independência $(p=0,000)$ (Tabela 3). 
Tabela 3 - Distribuição dos escores médios dos domínios de qualidade de vida dos enfermeiros das equipes de saúde da família da Macrorregião de Saúde do Triângulo do Sul-MG, segundo variáveis sociodemográficas, 2007

\begin{tabular}{|c|c|c|c|c|c|c|c|}
\hline \multirow[b]{2}{*}{ Variáveis $(n=90)$} & \multicolumn{7}{|c|}{ Domínios } \\
\hline & $\mathbf{n}$ & Físico & Psicológico & $\begin{array}{l}\text { Nível de inde- } \\
\text { pendência }\end{array}$ & $\begin{array}{c}\text { Relações } \\
\text { sociais }\end{array}$ & Ambiente & $\begin{array}{l}\text { Aspectos } \\
\text { espirituais }\end{array}$ \\
\hline \multicolumn{8}{|l|}{ Sexo } \\
\hline Feminino & 83 & 14,1 & 15,3 & 17,0 & 16,0 & 14,1 & 16,8 \\
\hline Masculino & 7 & 13,6 & 16,3 & 17,1 & 17,6 & 15,2 & 16,2 \\
\hline \multicolumn{8}{|l|}{ Faixa etária (anos) } \\
\hline $20+30$ & 62 & 14,2 & 15,5 & 17,2 & 16,6 & 14,4 & 17,1 \\
\hline $30+40$ & 17 & 13,5 & 14,9 & 16,3 & 15,5 & 13,5 & 16,1 \\
\hline $40+50$ & 7 & 13,7 & 15,3 & 16,6 & 14,3 & 14,4 & 16,1 \\
\hline \multicolumn{8}{|l|}{ Estado civil } \\
\hline Solteiro(a) & 52 & 14,4 & $15,4^{\mathrm{b}}$ & $16,9^{\mathrm{b}}$ & $16,3^{b}$ & $14,2^{b}$ & 16,7 \\
\hline Casado(a)/Amasiado(a) & 30 & 13,8 & $15,8^{\mathrm{a}}$ & $17,4^{\mathrm{a}}$ & $16,7^{\mathrm{a}}$ & $14,6^{\mathrm{a}}$ & 17,0 \\
\hline Divorciado(a) & 7 & 12,8 & $13,5^{\mathrm{b}}$ & $15,6^{b}$ & $13,4^{\mathrm{b}}$ & $12,5^{b}$ & 16,5 \\
\hline Valor $p$ & & & $0,013^{*}$ & $0,021^{*}$ & $0,000^{*}$ & $0,022^{*}$ & \\
\hline \multicolumn{8}{|l|}{ Escolaridade } \\
\hline Graduação & 36 & 14,2 & 15,4 & 17,0 & 15,9 & 14,0 & 16,7 \\
\hline Especialização & 54 & 14,0 & 15,3 & 17,0 & 16,3 & 14,3 & 16,8 \\
\hline \multicolumn{8}{|l|}{ Estado de saúde } \\
\hline "Muito bom ou bom" & 81 & $14,3^{\mathrm{d}}$ & $15,6^{d}$ & $17,2^{\mathrm{d}}$ & 16,4 & 14,2 & 16,9 \\
\hline "Nem ruim, nem bom" & 2 & $14,5^{d}$ & $15,9^{d}$ & $16,5^{\mathrm{d}}$ & 13,8 & 11,6 & 16,5 \\
\hline "Fraco ou Muito ruim" & 5 & $10,8^{c}$ & $12,4^{c}$ & $14,2^{\mathrm{c}}$ & 14,0 & 12,9 & 15,8 \\
\hline Valor $p$ & & $0,000^{*}$ & $0,002^{*}$ & $0,000^{*}$ & & & \\
\hline
\end{tabular}

${ }^{*}$ ANOVA-F - diferenças estatisticamente significativas a $>$; $c<<$.

\section{DISCUSSÃO}

Observou-se que os resultados obtidos neste estudo, em relação ao perfil dos enfermeiros, corroboram com os achados de outros estudos, ${ }^{3,5,11-13} \mathrm{em}$ que a enfermagem é exercida, principalmente, por mulheres. Os resultados desta pesquisa também confirmam os resultados encontrados no Brasil, ou seja, 90,9\% dos enfermeiros que atuam na ESF são compostos pelo sexo feminino. ${ }^{14} \mathrm{Na}$ enfermagem, esta característica histórica parece estar ligada à própria essência da profissão: o cuidado. $\mathrm{O}$ ato de cuidar sempre foi associado à figura feminina. ${ }^{15}$

A faixa etária encontrada para os enfermeiros das equipes de saúde da família do Triângulo Sul confirmam pesquisas realizadas em dez grandes centros urbanos, onde os enfermeiros da ESF são, na maioria, jovens, com idade abaixo de 30 anos. ${ }^{16}$ Em contrapartida, estudo nacional obteve que a maioria dos enfermeiros estava na faixa etária acima dos 30 anos, com uma média de 34 anos; valores superiores aos encontrados neste estudo. ${ }^{14}$

Os dados demonstraram que, no caso do Sul do Triângulo Mineiro, a ESF parece constituir-se uma opção mais efetiva de trabalho para profis- sionais jovens, com pouco tempo de experiência ou recém-formados. Tal constatação pode ser relacionada ao maior conhecimento dos profissionais com menor tempo de formação acerca das bases e princípios da ESF, devido à inserção, relativamente recente, deste conteúdo, nos currículos dos cursos de graduação em enfermagem. Estudos realizados na Saúde da família de Curitiba-PR ${ }^{12}$ e em âmbito nacional ${ }^{14}$ apontaram que os enfermeiros eram formados há menos de 15 anos.

Observou-se a predominância dos enfermeiros que não possuíam companheiro, fato semelhante aos resultados encontrados no município de Curitiba-PR. ${ }^{12}$ Estas características do perfil podem estar relacionadas ao fato de a população estudada ser, predominantemente, jovem. Ademais, as mulheres que são casadas, apresentam taxas mais baixas de inserção no mercado de trabalho, seja por discriminação ou por encargos familiares ou domésticos. ${ }^{17}$

A maioria dos participantes classificou sua saúde atual como "boa" ou "muito boa" (92\%), o que explica, de certa forma, porque $66,7 \%$ negaram possuir qualquer problema de saúde. Estudo com os trabalhadores da Saúde da Família de um 
município de Santa Catarina também constatou que os enfermeiros estavam satisfeitos com a própria saúde. ${ }^{11}$

Em relação à escolaridade, os dados deste estudo corroboram outras evidências. ${ }^{3,12} \mathrm{~A}$ busca por cursos de especialização pode ser explicada, por um lado, pela maior seletividade do mercado de trabalho e a esperança de aumento salarial. Por outro, a necessidade de ter um perfil diferenciado para o trabalho na ESF pode ter contribuído para este movimento, além da possibilidade de trabalhar no ensino de graduação, que passou a ser um mercado em expansão nos últimos anos. ${ }^{18}$

O estado conjugal influenciou os domínios psicológico, nível de independência, relações sociais e ambiente; os indivíduos que possuem companheiro apresentaram melhores escores de QV se comparados aos que não possuem.

Com relação ao domínio psicológico, que inclui as facetas sentimentos positivos e negativos, cognição, autoestima e satisfação com a imagem corporal e a aparência, o resultado confirma corrobora o estudo que destacou que possuir uma relação estável se associa a uma melhor percepção de QV neste domínio. ${ }^{19}$ A manutenção de uma relação de casal pode significar, geralmente, uma gratificação na vida. $\mathrm{O}$ afeto entre o casal gera sentimentos e pensamentos positivos, melhor autoestima e, em geral, os problemas próprios da vida são, muitas vezes, melhor administrados, se são compartilhados com o parceiro.

Em relação ao nível de independência, que destaca itens como mobilidade, atividades da vida cotidiana, capacidade para o trabalho e dependência de medicação/tratamento, a melhor percepção da QV dos enfermeiros com companheiro pode estar associada à relação de apoio do casal nas atividades da vida cotidiana, como criação dos filhos e tarefas domésticas. ${ }^{20}$

No domínio relações sociais, que possui facetas que avaliam as relações pessoais, o suporte social e a atividade sexual, os resultados concordam com a literatura científica quando sugerem que a estabilidade de um relacionamento tende a melhorar a satisfação com as relações familiares e com a atividade sexual. ${ }^{21}$ Uma relação de casal estável é elemento importante de apoio social. A ausência de suporte social, gerado pela individualidade, pelo individualismo, pode afetar o espaço das relações interpessoais e o da própria interioridade. Por outro lado, o fato de manter uma relação de casal permite viver e desfrutar da sexualidade de maneira mais estável. ${ }^{19}$
Quanto ao ambiente, que inclui as facetas segurança física e proteção, moradia, recursos financeiros, disponibilidade e qualidade dos cuidados de saúde e sociais, oportunidades de adquirir novas informações e habilidades, lazer, ambiente físico e transporte, o compartilhar a vida com um parceiro significa, muitas vezes, sensação de segurança, tanto no lar como no plano pessoal e econômico. ${ }^{19}$

Encontrou-se influência da autopercepção do nível de saúde em relação aos domínios físico, psicológico e nível de independência. O relato de baixo nível de saúde se relacionou com escores mais baixos de QV.

Com relação ao domínio físico, que abrange as facetas acerca de dor e desconforto, energia e fadiga, e sono e repouso, estudos evidenciam que o adoecimento físico e psíquico dos indivíduos devido à sobrecarga relacionada às condições inadequadas do ambiente e organização do trabalho é um aspecto evidente e vivido pelos profissionais de enfermagem..$^{22}$ Como já visto, soma-se a essas condições, o desgaste relacionado ao acúmulo de várias jornadas de trabalho, decorrente da diversidade de papéis sociais associadas ao sexo feminino. $\mathrm{O}$ excesso de trabalho origina cansaço e dores, o que torna os trabalhadores fisicamente mais desgastados. ${ }^{19}$

No âmbito psicológico, percebe-se que o modelo construído e determinado historicamente de devotamento, abnegação e dedicação ao cuidado com o próximo levam os profissionais a criar estratégias de defesa contra a dor e a , abandonar o autocuidado, o que leva ao adoecimento. ${ }^{22} \mathrm{~A}$ existência de uma doença com possível grau de prejuízo da qualidade da assistência pode gerar nos profissionais sentimentos de culpa e impotência diante das exigências do trabalho.

Em relação ao nível de independência, de modo geral, é de se esperar que a instalação de algum agravo à saúde promova comprometimento da mobilidade, das atividades do cotidiano, inclusive, da capacidade para o trabalho. Diante desse quadro, e observando a baixa avaliação da faceta dependência de medicação ou tratamento, se pode suspeitar que os profissionais possam utilizar-se de automedicação como estratégia de fuga e sublimação para sintomas de morbidades, possivelmente instaladas e não diagnosticadas.

\section{CONCLUSÃO}

Quanto à caracterização sociodemográfica dos participantes deste estudo, observou-se maioria de jovens do sexo feminino e solteira. 
Obteve-se maior prevalência dos indivíduos que possuíam especialização, realizavam curso de atualização e que avaliaram positivamente o próprio estado de saúde.

Os resultados mostram mostraram pouco impacto negativo ou ausência de impacto negativo nos componentes dos domínios na avaliação da QV dos enfermeiros.

Percebe-se que apesar de os enfermeiros da Macrorregião de Saúde do Triângulo Sul apresentarem escores médios condizentes com uma QV satisfatória, pôde-se observar que determinados condicionantes das variáveis sociodemográficas estado civil e estado de saúde, trazem prejuízos à QV dos enfermeiros da ESF, afetando os domínios psicológico e nível de independência, seguidos pelos domínios físico, relações sociais e ambiente.

Estes dados denotam a necessidade da definição de políticas públicas voltadas para a atenção à saúde profissional, uma vez que o relato de autopercepção insatisfatória com o estado de saúde interferiu negativamente na QV.

É preciso considerar a complexidade da temática $Q V$, pois ela reflete as condições e os valores individuais, que podem modificar-se de acordo com as características de determinado momento da vida das pessoas. Contudo, a análise dos fatores sociodemográficos associados à $\mathrm{QV}$ fornece subsídios necessários para a implementação de mudanças nas condições de vida e trabalho, reduzindo a distância entre as expectativas pessoais e a realidade de trabalho desses profissionais. Ações que contribuam no desenvolvimento da QV dos enfermeiros das equipes de saúde da família são importantes e necessárias, considerando o fato de a QV desses profissionais influenciar fortemente a qualidade da assistência prestada.

\section{REFERÊNCIAS}

1. Ministério da Saúde (BR), Departamento de Ações Programáticas Estratégicas. Manual de hipertensão arterial e diabetes mellitus. Plano de reorganização da atenção à hipertensão arterial e ao diabetes mellitus: hipertensão arterial e diabetes mellitus. Brasília (DF): MS; 2001.

2. Bittencourt ZZLC, Alves Filho G, Mazzali M, Santos NR. Qualidade de vida em transplantados renais: importância do enxerto funcionante. Rev Saúde Pública. 2004 Out; 38(5):732-4.

3. Minayo MCS, Hartz ZMA, Buss PM. Qualidade de vida e saúde: um debate necessário. Ciênc Saúde Colet. 2000 Jan-Jun; 5(1):7-18.
4. Campos JF, David HMSL. Abordagens e mensuração da qualidade de vida no trabalho de enfermagem: produção científica. Rev Enferm UERJ. 2007 OutDez; 15(4):584-9.

5. Canesqui AM, Espinelli MAS. Saúde da família no Estado de Mato Grosso, Brasil: perfis e julgamentos dos médicos e enfermeiros. Cad Saúde Pública. 2006 Set; 22(9):1881-92.

6. Miranzi SSC, Ferreira FS, Iwamoto HH, Pereira GA, Miranzi MAS. Qualidade de vida de indivíduos com diabetes mellitus e hipertensão acompanhados por uma equipe de saúde da família. Texto Contexto Enferm. 2008 Out-Dez; 17(4):672-9.

7. Seidl EMF, Zannon, CMLC. Qualidade de vida e saúde: aspectos conceituais e metodológicos. Cad Saúde Pública. 2004 Mar-Abr; 20(2):580-8.

8. The whoqol group. The World Health Organization quality of life assessment (WHOQOL): position paper from the World Health Organization. Soc Sci Med. 1995 Nov; 41(10):1403-9.

9. Ximenes Neto FRG, Sampaio JJC. Gerentes de território na estratégia saúde da família: análise e perfil de necessidades de qualificação. Rev Bras Enferm. 2007 Nov-Dez; 60(6):687-95.

10. Fleck MPA, Lousada S, Xavier M, Chachamovich E, Vieira G, Santos L, et al. Aplicação da versão em português do instrumento de avaliação de qualidade de vida da OMS (WHOQOL-100). Rev Saúde Pública. 1999 Abr; 33(2): 198-205.

11. Gessner CLS. Qualidade de vida das equipes de saúde da família do município de Timbó-SC [dissertação]. Itajaí (SC): Universidade do Vale do Itajaí, Programa de Mestrado Profissionalizante em Saúde e Gestão do Trabalho; 2006.

12. Gil CRR. Práticas profissionais em saúde da família: expressões de um cotidiano em construção [tese]. Rio de Janeiro (RJ): Escola Nacional de Saúde Pública Sergio Arouca; 2006.

13. Santos RMA, Beresin RA. Qualidade de vida dos enfermeiros do centro cirúrgico. Einstein. 2009; 7(2):152-8.

14. Machado MH, organizadora. Perfil dos médicos e enfermeiros do programa de saúde da família no Brasil: relatório final. Brasília (DF): Ministério da Saúde; 2000.

15. Souza ML, Sartor VVB, Padilha MICS, Prado ML. O Cuidado em enfermagem: uma aproximação teórica. Texto e Contexto Enferm. 2005 Abr-Jun; 14(2):266-70.

16. EscorelS, Giovanella L, Mendonça MHM, Magalhães $\mathrm{R}$, Senna MCM. Avaliação da implementação do programa de saúde da família em dez grandes centros urbanos: síntese dos principais resultados. Brasília (DF): Ministério da Saúde; 2002.

17. Cárdenas AMC. Qualidade de vida de mulheres de uma comunidade de baixa renda: estudo comparativo 
entre mulheres com trabalho doméstico e trabalho remunerado [tese]. São Paulo (SP): Universidade de São Paulo. Escola de Enfermagem; 2004.

18. Pierantoni CR, Machado MH, Campos FE, Ferreira JR, Abramzón MC, organizadores. Trabalho e educação em saúde no Mercosul. Brasília (DF): Ministério da Saúde, Rio de Janeiro: Europa; 2008.

19. Barrientos LA, Suazo SV. Fatores associados a qualidade de vida de enfermeiras hospitalares chilenas. Rev Latino-am Enfermagem. 2007 MaioJun; 15(3):480-6.
20. Spindola T, Santos RS. Mulher e trabalho: a história de vida de mães trabalhadoras de enfermagem. Rev Latino-am Enfermagem. 2003 Set-Out; 11(5):593-600.

21. Salles EP. Qualidade de vida do auxiliar e técnico de enfermagem em UTIs [dissertação]. Goiania (GO): Universidade Federal de Goiás. Programa de PósGraduação em Enfermagem; 2005.

22. Elias MA, Navarro VL. A relação entre o trabalho, a saúde e as condições de vida: negatividade e positividade no trabalho das profissionais de enfermagem de um hospital escola. Rev Latino-am Enfermagem. 2006 Jul-Ago; 14(4):517-25. 